\title{
Clinical management of atrial fibrillation: early interventions, observation, and structured follow-up reduce hospitalizations
}

\author{
Alberto Conti MD ${ }^{a, *}$, Erica Canuti MD ${ }^{a}$, Yuri Mariannini MD ${ }^{a}$, Gabriele Viviani MD ${ }^{a}$, \\ Claudio Poggioni MD ${ }^{a}$, Vanessa Boni MD ${ }^{a}$, Riccardo Pini MD ${ }^{a}$, Simone Vanni MD ${ }^{b}$, \\ Luigi Padeletti MDc, Gian Franco Gensini MD ${ }^{c}$
}

\author{
${ }^{a}$ Emergency Medicine, Department of Critical Care Medicine and Surgery, Careggi University Hospital, Florence, Italy \\ ${ }^{\mathrm{b}}$ Emergency Department, Careggi University Hospital, Florence, Italy \\ ${ }^{\mathrm{c}}$ Department of Cardiology, Careggi University Hospital, Florence, Italy
}

Received 25 November 2011; revised 8 March 2012; accepted 17 April 2012

\begin{abstract}
Background: Novel facilities such as an intensive observation unit and an outpatient clinic could result in improving management of patients presenting with atrial fibrillation (AF).

Methods: This observational study enrolled 3475 patients. Group 1 (1120 patients; years 2004-2005) was managed with standard approach; group 2 (992 patients; years 2006-2007) was managed with additional intensive observation; group 3 (1363 patients; years 2008-2009) was managed with additional intensive observation and outpatient clinic. Primary end point was admission to hospital. Secondary end points included modalities of rhythm conversion and administration of class IC vs class III antiarrhythmic drugs in patients with AF lasting less than 48 hours.

Results: Lack of rhythm control, comorbidities, diabetes, and age were independent predictors of hospitalization. Admissions significantly decreased from group $1(50 \%)$ to $2(38 \%)$ and to $3(24 \%)(P<$ $.001)$. Interestingly, more than a quarter of patients in group 3 were referred to the outpatient clinic for short-term follow-up, eventually avoiding admission. Patients with AF lasting less than 48 hours $(\mathrm{n}=$ $2189)$ and without structural heart disease $(n=1685)$ achieved sinus rhythm in $89 \%$ of cases and were discharged. In these patients, early administration of antiarrhythmic drugs of class IC and III gained sinus rhythm in $80 \%$ and $20 \%$, respectively $(P<.001)$. Spontaneous conversion occurred in $26 \%$; electrical, 17\%; and pharmacological, $57 \%$.

Conclusions: In patients with AF, beyond the standard approach, the novel organization with an additional intensive observation unit for early pharmacological interventions and an outpatient clinic for elective treatment and short-term follow-up significantly reduced admission irrespective of independent predictors of hospitalizations. Patients without structural heart disease treated with antiarrhythmic drugs achieved sinus rhythm in $89 \%$ of cases, mostly with class IC drugs.

(C) 2012 Published by Elsevier Inc.
\end{abstract}

* Corresponding author.

E-mail address: aaaconti@hotmail.com (A. Conti). 


\section{Introduction}

The prevalence of atrial fibrillation (AF) has substantially increased during the last 2 decades because of the aging of the population, the increasing prevalence of chronic heart disease, and the more frequent diagnoses by monitoring devices [1]. At the same time, a substantial increase in the number of hospitalizations for AF has been observed $[2,3]$. Indeed, a report of the American Heart Association showed hospitalizations, with $\mathrm{AF}$ as the first-listed diagnosis, increased by $34 \%$ from 1996 to 2001 [4] and by $13 \%$ in the past 2 decades in Europe [5]. This trend is related to the changing practice pattern with AF management [6] rather than changes over time in the distribution of age, sex, and comorbidities [3,7]. Because the development of AF is age related and the age-adjusted incidence of $\mathrm{AF}$ is also rising, the incidence and prevalence of this condition are expected to increase in the next few decades [2]. As a consequence, $\mathrm{AF}$ is a costly public health problem, with hospitalizations as the primary cost driver [8] both in North America and in Europe $[9,10]$. Therefore, the aim of the present study was to assess if, beyond the standard approach, the novel organization with an additional intensive observation unit for early pharmacological interventions and an outpatient clinic for elective treatment and follow-up could avoid admissions irrespective of independent predictors of hospitalizations.

\section{Methods}

This observational study considered for enrolment $\mathrm{AF}$ patients presenting to the emergency department (ED) of a tertiary care teaching hospital: Careggi University Hospital, Florence, Italy. Facilities for triage of patients with suspected dysrhythmia included an observation unit with monitorequipped beds and dedicated personnel such as cardiologists on call and residents. Coded diagnoses are inserted into an electronic medical chart, allowing easy identification of all patients with a diagnosis of interest. Data were collected from January 2004 to January 2010.

\subsection{Patient selection}

Consecutive patients complaining of palpitations or general malaise were considered, and those with AF documented by an electrocardiogram (ECG) were evaluated in the ED and enrolled in the study. Exclusion criteria were age less than 18 years; Killip Class 2 or more; New York Heart Association functional class III or more; clinically detectable ongoing complications such as acute coronary syndrome, stroke, and pulmonary or peripheral embolism; and hemodynamic instability. Three groups of patients were analyzed: group 1, patients evaluated in the years 2004-2005; group 2, patients evaluated in the years 2006-2007; and group 3, patients evaluated in the years 2008-2009. Along these years, in the ED of our hospital, the clinical management of AF changed because an observation unit for early pharmacological interventions and an outpatients clinic for structured follow-up were added to the standard approach. Thus, group 1 was managed with standard approach; group 2 was managed with an additional intensive observation; and group 3 was managed with both an additional intensive observation and an outpatient clinic. All patients gave informed consent to participate in the study and publication of data processing.

The study was conducted according to good clinical practice and the Helsinki principles. The institutional review board approved the protocol.

\subsection{Definition of subtype of AF}

Paroxysmal AF was defined by electrocardiographic evidence of AF followed by subsequent ECG showing sinus rhythm or clinical documentation by a physician of paroxysmal AF; persistent $\mathrm{AF}$ was defined when lasting longer than 7 days or when terminated by therapeutic treatment; permanent $\mathrm{AF}$ (or chronic $\mathrm{AF}$ ) was defined by the presence of long-lasting (up to year) serial ECGs showing only AF and no interim evidence of sinus rhythm; solitary or lone $\mathrm{AF}$ was defined by $\mathrm{AF}$ in the absence of overt cardiovascular disease or precipitating illness [5,11].

\subsection{Definition of structural heart disease}

Known existing coronary artery disease was defined by angiographic findings of lesions greater than $50 \%$ in any coronary artery or a history of acute coronary syndrome or revascularization. The definition of acute or stable coronary syndromes was derived from the European Society of Cardiology and American Heart Association guidelines [12-14]. Cardiomyopathy was defined by the presence of a reported physician diagnosis or echocardiographic confirmation of abnormal dilated left or right ventricles; hypertrophic cardiomyopathy was defined by the presence of severe left ventricular hypertrophy.

\subsection{Definition of moderate to severe comorbidities}

Hemodynamic instability was defined by the presence of systolic blood pressure less than $100 \mathrm{~mm} \mathrm{Hg}$ and/or poor skin signs and/or altered level of consciousness and/or severe shortness of breath or pulmonary edema (Advanced Life Support Protocols, Adult Hemodynamic Instability Definitions, Policy Reference No. 602, San Luis Obispo County EMS Agency, ALS Treatment Protocols 2007 [Rev. 11/1/10]; http://www.sloemsa.org/files/602.pdf) [15]. Severe vascular disease included carotid and peripheral artery disease referring to the presence of a grade of obstruction greater than $70 \%$ in any vessel. Peripheral arterial disease also referred to a history of intermittent 
claudication or reported physician diagnosis documented by imaging.

Abnormal kidney function was defined as the presence of serum creatinine of at least $2.3 \mathrm{mg} / \mathrm{dL}$. Abnormal liver function was defined as chronic hepatic disease (eg, cirrhosis) or biochemical evidence of significant hepatic derangement (eg, bilirubin $>2 \times$ upper limit of normal, in association with aspartate aminotransferase/alanine aminotransferase/alkaline phosphatase $>3 \times$ upper limit normal) $[4,6]$. Severe systemic hypertension was defined by a reported diagnosis of classification of hypertension in the third class of the World Health Organization. Overall cardiovascular comorbidities were eventually defined as the composite of known existing coronary artery disease, valvular heart disease, and carotid and peripheral artery disease. Moderate to severe chronic obstructive pulmonary disease and dysthyroidism were considered in the presence of documented medical records.

\subsection{Management of patients and study protocol}

During the time of triage to the ED, a research nurse detected vital signs and ECG monitoring on admission (systolic and diastolic blood pressure, heart rate, ECG monitoring, oxygen saturation, and breaths per minute) and prospectively recorded variables used for the risk stratification and information obtained from patients, relatives, caregivers, or emergency ambulance crews. The onset of AF was clinically determined on the basis of reported symptoms. During the visit, the resident checked the correctness of the findings and integrated them with data obtained from patients and from previous hospitals' freely accessed folders available on the hospital network. Eventually, the resident confirmed the diagnosis obtained by ECG monitoring of the emergency ambulance crews who are designed to provide a clinical workplace with the maximum mobility. At the time of the first contact with the resident, all patients underwent evaluation with medical history, physical examination, initial 12-lead ECG, plasma level of troponin I, and blood gas analysis. Blood tests, chest radiographs, and serial ECGs were performed according to the clinical conditions during the first-line 6-hour period inside the ED. All the patients with unclear clinical history and unclear pattern of clinical presentation were subjected to urgent echocardiography.

Patients with AF onset of less than 48 hours and without moderate to severe structural heart disease were considered eligible for rhythm control. They received immediately intravenous bolus (in a few minutes) of flecainide ( $2 \mathrm{mg} /$ $\mathrm{kg})$, propafenone $(2 \mathrm{mg} / \mathrm{kg})$, or amiodarone $(5 \mathrm{mg} / \mathrm{kg})$ [5,11,16-19]. The chart of time to treatment is shown in Fig. 1. Patients with unclear history of known existing structural heart disease or patients who were considered as needing further evaluation (echocardiography, chest radiography, serial troponin I) before undergoing pharmacological treatment were submitted to intensive observation. All the

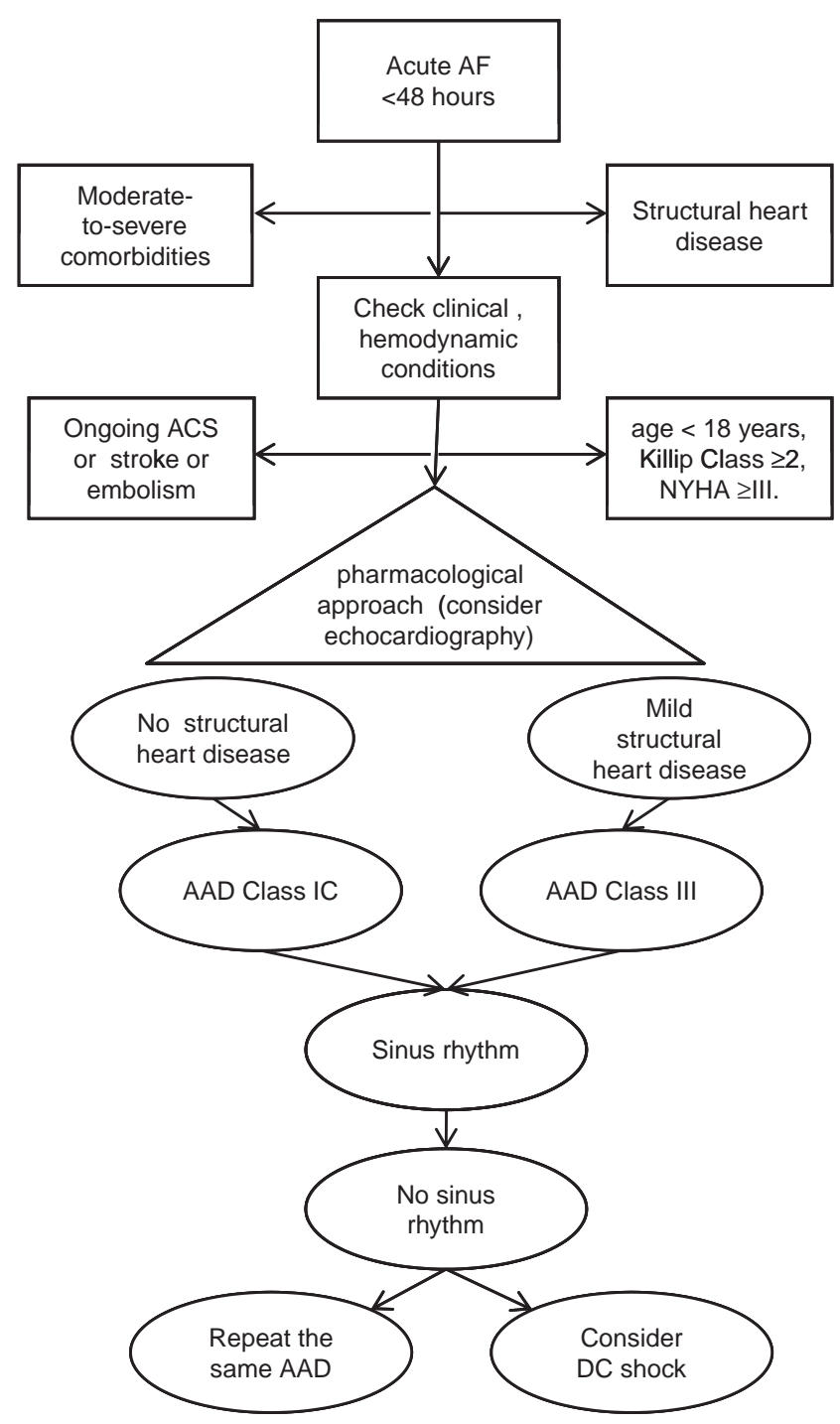

Fig. 1 The chart of time to treatment in patients presenting with AF lasting less than 48 hours. Class ADD, Vaughan-Williams class of antiarrhythmic drugs.

patients who did not recover sinus rhythm were considered for a second dose of drugs previously given, eventually followed by oral administration up to 24 hours of flecainide $(2 \mathrm{mg} / \mathrm{kg}$ plus $100 \mathrm{mg}$ twice a day), propafenone ( $2 \mathrm{mg} / \mathrm{kg}$ plus $150 \mathrm{mg}$ thrice a day), or amiodarone ( $5 \mathrm{mg} / \mathrm{kg}$ plus $200 \mathrm{mg}$ twice a day). In patients with acute AF, those who did not recover sinus rhythm after the first-line approach (inside the ED) and the second-line approach (inside the intensive observation) were submitted to emergency cardioversion, eventually after the 24-hour management at least, to not exceed 48 hours and to prevent systemic embolism. After direct current cardioversion, patients were observed for a period of 3 to 6 hours to promptly detect adverse events. Patients with AF lasting more than 48 hours were submitted to oral anticoagulation and elective direct current cardioversion. Patients who were discharged to follow-up in the outpatient clinic were managed with anticoagulation. Inside the observation unit, they started 
Table 1 Baseline clinical characteristics of patients with AF enrolled in the study $(\mathrm{N}=3475)$

\begin{tabular}{|c|c|c|c|c|c|c|c|c|}
\hline Clinical characteristics & $\begin{array}{l}\text { Total } \\
(\mathrm{N}=3475)\end{array}$ & $\begin{array}{l}\text { Group 1 } \\
(\mathrm{n}=1120)\end{array}$ & $\begin{array}{l}P \\
1 \text { vs } 2 \\
\end{array}$ & $\begin{array}{l}\text { Group } 2 \\
(\mathrm{n}=992)\end{array}$ & $\begin{array}{l}P \\
2 \text { vs } 3\end{array}$ & $\begin{array}{l}\text { Group } 3 \\
(\mathrm{n}=1363)\end{array}$ & $\begin{array}{l}P \\
3 \text { vs } 1 \\
\end{array}$ & $P$ ANOVA \\
\hline Age (y SD) & $70 \pm 113$ & $70 \pm 13$ & NS & $70 \pm 13$ & NS & $71 \pm 12$ & NS & .053 \\
\hline Gender (male) & $1695(49 \%)$ & $519(46 \%)$ & NS & $491(50 \%)$ & NS & $685(50 \%)$ & NS & .131 \\
\hline Hypertension & $1739(50 \%)$ & $591(53 \%)$ & NS & $496(50 \%)$ & NS & $652(48 \%)$ & NS & .050 \\
\hline Diabetes mellitus & $288(8 \%)$ & $88(8 \%)$ & NS & $85(9 \%)$ & NS & $115(8 \%)$ & NS & .812 \\
\hline Comorbidities & $1512(44 \%)$ & $530(47 \%)$ & NS & $419(42 \%)$ & NS & $563(41 \%)$ & .009 & .007 \\
\hline $\mathrm{AF} \geq 48 \mathrm{~h}$ & $1286(37 \%)$ & $506(45 \%)$ & $<.001$ & $364(37 \%)$ & .006 & $416(31 \%)$ & $<.001$ & $<.001$ \\
\hline Lack of rhythm control & $1414(41 \%)$ & $508(45 \%)$ & NS & $427(43 \%)$ & $<.001$ & $479(35 \%)$ & $<.001$ & $<.001$ \\
\hline Admission rate & $1262(34 \%)$ & $557(50 \%)$ & $<.001$ & $379(38 \%)$ & $<.001$ & $326(24 \%)$ & $<.001$ & $<.001$ \\
\hline
\end{tabular}

NS, not significant.

both subcutaneous low-molecular weight heparin and oral warfarin. Moreover, during the observation period, they received the education that they needed to know in how to take anticoagulation both with low-molecular weight heparin and with warfarin until they gained a target value of international normalized ratio greater than 2 .

Because no standard approach is currently being used in our setting, no randomization was used; and the therapeutic approach was at the discretion of the physician on duty. Patients with AF lasting more than 48 hours were considered not eligible for early rhythm conversion $[5,11]$. Eventually, they were subjected to elective electrical conversion (previous transesophageal echocardiography or after 3 weeks of anticoagulation treatment). Residents and senior doctors ran our observation unit; cardiologists were on call. Patients were expected to stay in observation for no more than 24 hours. Patients were sent to the outpatients clinic for short-term evaluation in the presence of increased AF symptoms burden and with limitations of drug therapy. Patients were admitted in the presence of moderate to severe comorbidities and unstable hemodynamic conditions. Patients with spontaneous cardioversion were considered those who gained sinus rhythm without any pharmacological treatment.

\subsection{End point}

The primary end point was admission to hospital. Secondary end points included modalities of rhythm conver- sion and administration of class IC vs class III antiarrhythmic drugs in patients with AF lasting less than 48 hours.

\subsection{Statistical analysis}

Summary data are expressed as mean \pm SD. Statistical comparisons of demographic and clinical features among the 3 groups were performed using $\chi^{2}$ test followed by the Bonferroni test for nonparametric variables as required. Analysis of variance followed by Scheffe test as appropriate was used for parametric variables. Univariate and multivariate logistic regression analyses were used to define independent predictors of hospitalization. $P$ values are 2 -sided. A $P$ value less than .05 was considered statistically significant. Calculations were performed with use of version 17, SPSS statistical package (SPSS Inc, Chicago, Illinois).

\section{Results}

\subsection{Patients enrolled in the study}

From January 2004 to January 2010, 3475 AF patients presenting to the ED (1.1\% of total visits per year) were enrolled and stratified into 3groups: group 1 (years 20042005) included 1120 patients, group 2 (years 2006-2007) included 992 patients, and group 3 (years 2008-2009) included 1363 patients.

Table 2 Association between clinical variables and primary end point at univariate and multivariate analysis

\begin{tabular}{|c|c|c|c|c|c|c|}
\hline & \multicolumn{3}{|c|}{ Univariate analysis } & \multicolumn{3}{|c|}{ Multivariate analysis } \\
\hline & OR & $95 \% \mathrm{CI}$ & $P$ & OR & $95 \%$ CI & $P$ \\
\hline Lack of rhythm control & 9.837 & 8.409-11.592 & $<.001$ & 8.653 & $7.343-10.197$ & $<.001$ \\
\hline Diabetes mellitus & 2.421 & $2.102-2.789$ & $<.001$ & 1.864 & $1.581-2.197$ & $<.001$ \\
\hline Comorbidities & 2.156 & $1.692-2.749$ & $<.001$ & 1.700 & $1.277-2.263$ & $<.001$ \\
\hline Age & 1.034 & $1.028-1.041$ & $<.001$ & 1.016 & $1.009-1.023$ & $<.001$ \\
\hline Hypertension & 0.987 & $0.860-1.134$ & NS & - & - & - \\
\hline Male gender & 0.930 & $0.810-1.068$ & NS & - & - & - \\
\hline
\end{tabular}




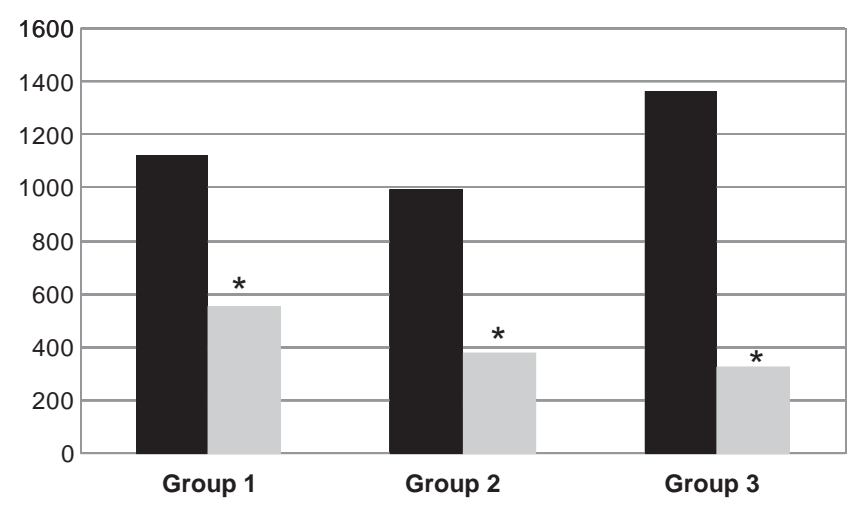

Fig. 2 Patients enrolled in groups 1, 2 and 3 (black bars); patients admitted (gray bars). ${ }^{*} P<.001$.

\subsection{Study population}

Baseline clinical characteristics of enrolled patients are shown in Table 1. In our population, approximately one-half of patients showed comorbidities and hypertension; onethird presented AF lasting more than 48 hours; mean age was 70 years. Nonetheless, in the prospective observational design of the study, no differences existed among clinical characteristics of the 3 groups of patients including mean age, gender, hypertension, and diabetes. Conversely, comorbidities were greater in the third group as compared to the first group; AFs lasting more than 48 hours were lower in the third group as compared to the other groups, as shown in Table 1. Overall, 1286 (27\%) patients had AF lasting more than 48 hours. Out of the 2189 remaining patients with AF lasting less than 48 hours and considered for cardioversion by immediate pharmacological treatment, 504 (14\%) patients had moderate to severe structural heart disease or moderate to severe comorbidities; they were not submitted to immediate pharmacological treatment. Thus, 1685 patients underwent the study protocol for pharmacological treatment.

At univariate and multivariate analysis, lack of rhythm control, diabetes mellitus, comorbidities, and age were independent predictors of hospitalization (Table 2). Overall admissions significantly decreased when the intensive observation unit and outpatient clinic were available, as follows: in group 1, admissions accounted for $50 \%$ patients; in group 2, admissions accounted for $36 \%$; and in group 3 , admissions accounted for $24 \%(P<.001$; Fig. 2$)$. Comorbid- ities $(\mathrm{n}=1512)$ included structural heart disease $(27 \%)$, thyroid dysfunction $(5 \%)$, respiratory diseases $(6 \%)$, life expectancy less than 6 months (4\%), and other chronic diseases $(9 \%$, Table 3$)$. Only chronic disease reduced from group 1 to group 3 (Table 3). Patients with AF lasting less than 48 hours $(\mathrm{n}=2189,63 \%)$ and without structural heart disease $(\mathrm{n}=1685,77 \%)$ eligible for immediate rhythm control by pharmacological approach achieved sinus rhythm in $89 \%$ ( $\mathrm{n}=$ 1504) of cases. Spontaneous rhythm conversion occurred in $26 \%$ of patients; electrical conversion, $17 \%$; and pharmacological conversion, 57\%. Among patients who underwent pharmacological rhythm conversion, class IC antiarrhythmic drugs gained sinus rhythm in $80 \%$ of treated patients, whereas class III drugs did it in $20 \%(P<.001$, Fig. 3), without differences among the 3 groups (Table 4). Major adverse effects related to pharmacologic conversion were as follows: 2 patients developed polymorphic ventricular tachycardia after the infusion of class IC antiarrhythmic drugs and before recovering sinus rhythm; 8 patients developed bradycardia and hypotension after recovering the sinus rhythm. Thus, all these patients were discharged in sinus rhythm. All the previous reported major adverse effects were monitored by observation without any additional treatment.

Of note, in our series, adherence to guidelines was confirmed by the most prevalent use of class IC antiarrhythmic drugs over class III in all groups of patients of the 3 different periods $(P<.001$, Table 4$)$. Overall, $60 \%$ of enrolled patients gained sinus rhythm, the most with pharmacological therapy $(\mathrm{n}=1127,33 \%)$. Spontaneous and electrical rhythm conversions increased from group 1 to group $3(P<.001)$, whereas pharmacological rhythm conversions decreased from group 1 to group $3(P=.0024)$; however, pharmacological conversion remained the most representative approach in all groups (Table 5).

Finally, in the years $2008-2009,27 \%(\mathrm{n}=359)$ of the patients of group 3 were subjected to the outpatient clinic within a mean period of 2 weeks after discharge from the ED. During the subspecialty consultation, a clinical examination and ECG were performed. Mean age of patients presenting to outpatient clinic was 70 years; lack of rhythm control and comorbidities accounted for a substantial portion: $30 \%$ $(n=109)$ and $36 \%(n=129)$, respectively. Only $8 \%(n=30)$ of patients had diabetes, and $70 \%(n=250)$ had sinus rhythm at discharge from the ED.

Table 3 Comorbidities and structural heart disease in the 3 groups of patients enrolled in the study (group 1, years 2004-2005; group 2, years 2006-2007; group 3, years 2007-2010)

\begin{tabular}{|c|c|c|c|c|c|c|c|c|}
\hline & Total 3475 & $\begin{array}{l}\text { Group } 1 \\
1120\end{array}$ & $\begin{array}{l}P \\
1 \text { vs } 2\end{array}$ & $\begin{array}{l}\text { Group } 2 \\
992\end{array}$ & $\begin{array}{l}P \\
2 \text { vs } 3\end{array}$ & $\begin{array}{l}\text { Group } 3 \\
1363\end{array}$ & $\begin{array}{l}P \\
1 \text { vs } 3\end{array}$ & $P$ ANOVA \\
\hline Pulmonary disease (n, \%) & $202(6 \%)$ & $61(5 \%)$ & NS & $61(6 \%)$ & NS & $80(6 \%)$ & NS & NS \\
\hline Thyroid dysfunction (n, \%) & $181(5 \%)$ & $65(5 \%)$ & NS & $47(5 \%)$ & NS & $69(5 \%)$ & NS & NS \\
\hline Life expectancy $\leq 6$ mo (n, \%) & $147(4 \%)$ & $42(3 \%)$ & NS & $42(4 \%)$ & NS & $63(5 \%)$ & NS & NS \\
\hline Other chronic disease $(\mathrm{n}, \%)$ & $297(9 \%)$ & $144(13 \%)$ & $<.001$ & $65(7 \%)$ & NS & $88(6 \%)$ & $<.001$ & $<.001$ \\
\hline Overall structural heart disease (n, \%) & $921(27 \%)$ & $303(37 \%)$ & NS & $283(28 \%)$ & NS & $335(24 \%)$ & NS & NS \\
\hline
\end{tabular}




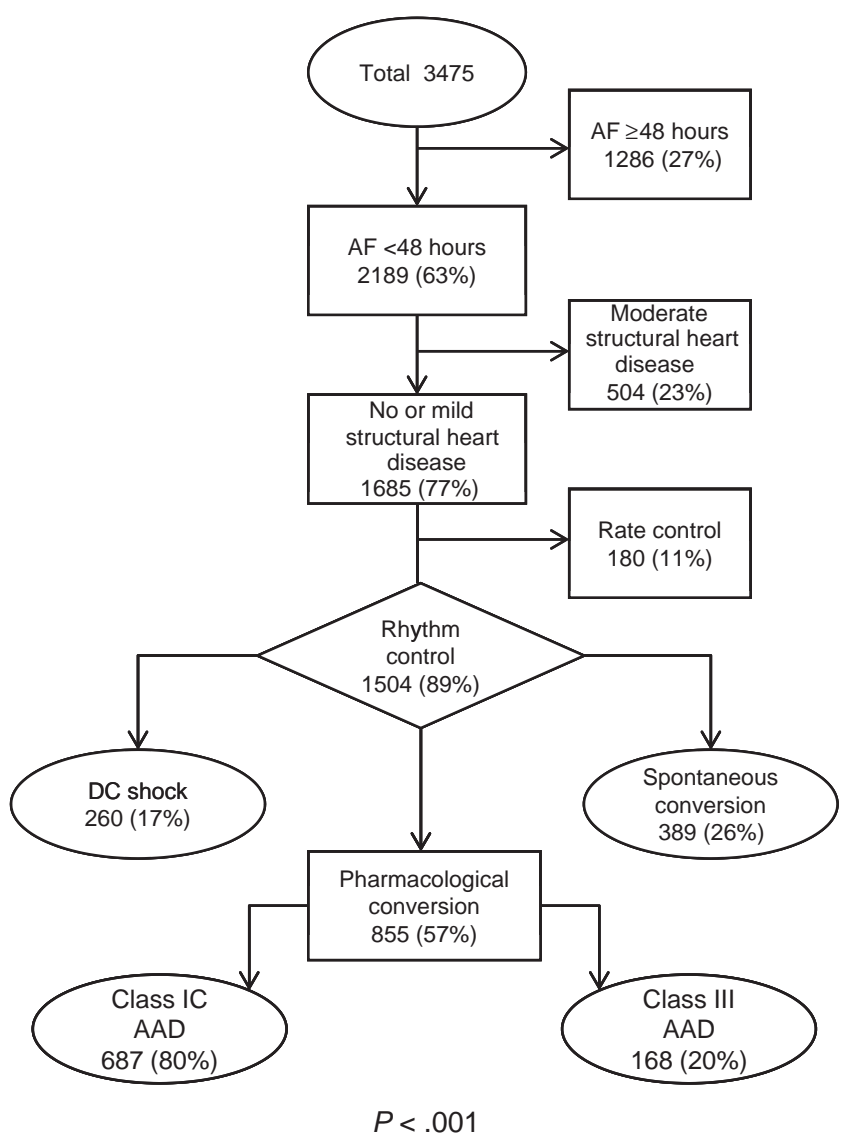

Fig. 3 Flow diagram of management of patients presenting with AF lasting less than 48 hours without structural heart disease and eligible for pharmacological conversion. Class IC AAD vs class III AAD, $P<.001$. Class ADD, Vaughan-Williams class of antiarrhythmic drugs.

\section{Discussion}

Our study demonstrates that patients presenting with $\mathrm{AF}$ lasting less than 48 hours had a lower rate of admission when the ED was set with novel planned facilities such as an intensive observation unit and an outpatient clinic. Indeed, admission accounted for $50 \%$ of patients managed without facilities, $38 \%$ of patients managed with an additional intensive observation unit, and $24 \%$ of patients managed

Table 4 Rates of conversion to sinus rhythm by different pharmacological treatment among the 3 groups of the study in patients with acute AF $(n=855)$

\begin{tabular}{llll}
\hline & Group 1 & Group 2 & Group 3 \\
\hline $\begin{array}{l}\text { Pharmacological } \\
\text { conversion (n) }\end{array}$ & 293 & 231 & 331 \\
Class IC AAD (n, \%) & $212(72 \%)$ & $195(84 \%)$ & $280(85 \%)$ \\
$P$ value & $<.001$ & $<.001$ & $<.001$ \\
Class III AAD (n, \%) & $81(28 \%)$ & $36(16 \%)$ & $51(15 \%)$ \\
\hline AAD, antiarrhythmic drug. & &
\end{tabular}

with both intensive observation unit and outpatient clinic $(P<.001)$. Admission was reduced irrespective of independent predictors of hospitalizations such as lack of rhythm control, diabetes, comorbidities, and age. Patients presenting with acute AF lasting less than 48 hours and without structural heart disease achieved sinus rhythm in $89 \%$ of cases, the most with class IC antiarrhythmic drugs, and were discharged. Class IC antiarrhythmic drugs' superiority over class III agents was confirmed in all the 3 periods of the study. Thus, emergency room physicians should be encouraged to use Class IC agents and to agree with the novel approach to acute AF.

More than a quarter of patients have been sent to the outpatient clinic for elective treatment and short-term followup, eventually avoiding admission.

Consistent with our findings, previous studies have reported observation units as a useful choice for improving the utilization of health care resources [20], especially for successful treatment of chest pain [21], asthma [22], and syncope [23] and eventually for treatment of AF, to reduce in-hospital stay [24]. Another study showed cost savings with the use of oral rate control agents, appropriate anticoagulation, and electrical cardioversion, with expedited referral to an outpatient AF clinic [25]. Indeed, also in our study, we observed a substantial percentage of pharmacological cardioversion among groups and a greater proportion of successful electrical cardioversions through the 6-year observation period, from $5 \%$ in group 1 , to $11 \%$ in group 2 , and to $15 \%$ in group 3 . Nonetheless, no strategy to pursue normal sinus rhythm including antiarrhythmic drug therapy, conversion, and ablation has definitely been shown to reduce the risk of stroke and hospitalization [4]. In our setting, physicians were strongly invited to consider pharmacological conversion as first-line treatment [11]. Hence, among patients who recovered sinus rhythm $(\mathrm{n}=2060)$, pharmacological approach was the most representative therapeutic strategy $(n=1127)$. Interestingly, when we consider patients with acute $\mathrm{AF}$ and without structural heart disease, adherence to guidelines was confirmed by the strong prevalent administration of class IC antiarrhythmic drugs over the administration of class III drugs in all groups $(P<.001)$. Previous trials demonstrated that class IC and class III antiarrhythmic drugs succeeded in decreasing admissions $[16,26]$. Additional evidence could result from new antiarrhythmic drugs with optimal safety profile for rapid conversion of $\mathrm{AF}$ also in patients with structural heart disease [27]. Patients with spontaneous cardioversion gained sinus rhythm without any pharmacological treatment. We compared the percentage of spontaneous cardioversion among groups along all the periods of the study. We observed a higher percentage of spontaneous cardioversion from group 1 to group 3 even if it did not gain statistical significance. We considered that this fact was due to increased awareness of the population about AF symptoms and that the early presentation to the ED was due to the fear of symptoms and of complications such as stroke. 
Table 5 Modalities of rhythm conversion in all patients with AF enrolled in the study and managed in the 3 different periods $(\mathrm{N}=3475)$

\begin{tabular}{|c|c|c|c|c|c|c|c|c|}
\hline & Total $\mathrm{N}=3475$ & $\begin{array}{l}\text { Group } 1 \\
\mathrm{n}=1120\end{array}$ & $\begin{array}{l}P \\
1 \text { vs } 2\end{array}$ & $\begin{array}{l}\text { Group } 2 \\
\mathrm{n}=992\end{array}$ & $\begin{array}{l}P \\
2 \text { vs } 3\end{array}$ & $\begin{array}{l}\text { Group } 3 \\
\mathrm{n}=1363\end{array}$ & $\begin{array}{l}P \\
1 \text { vs } 3\end{array}$ & $P$ ANOVA \\
\hline Sinus rhythm $(\mathrm{n}, \%)$ & $2060(60 \%)$ & $611(55 \%)$ & NS & $565(57 \%)$ & $<.001$ & $884(65 \%)$ & $<.001$ & $<.001$ \\
\hline $\begin{array}{l}\text { Spontaneous } \\
\text { conversion }(\mathrm{n}, \%)\end{array}$ & $562(16 \%)$ & $144(13 \%)$ & NS & $145(15 \%)$ & $<.001$ & $273(20 \%)$ & $<.001$ & $<.001$ \\
\hline $\begin{array}{l}\text { Electrical } \\
\text { conversion }(\mathrm{n}, \%)\end{array}$ & $370(11 \%)$ & $60(5 \%)$ & $<0.001$ & $107(11 \%)$ & $<.0108$ & $203(15 \%)$ & $<.001$ & $<.001$ \\
\hline $\begin{array}{l}\text { Pharmacological } \\
\text { conversion }(\mathrm{n}, \%)\end{array}$ & $1127(33 \%)$ & $407(36 \%)$ & NS & $312(32 \%)$ & NS & $408(30 \%)$ & .021 & .0024 \\
\hline
\end{tabular}

Finally, in the present study, more than a quarter of patients of group 3, in the last 2-year period of the study, were evaluated in the outpatient clinic. In these patients, because of the presence of one-third of patients with both comorbidities and lack of rhythm control, admission was considered a real option without the outpatients clinic. Moreover, two-third of patients managed with oral ratecontrol agents were subjected to elective treatment and shortterm follow-up in the outpatients clinic to reduce frequency and severity of AF symptoms and further admissions. Results of follow-up could face future analysis. Although the criteria for submission to the outpatient clinic were not standardized and at the discretion of the emergency physician on duty, we consider this option a remarkable step for further decrease of hospital admission. Costs of providing outpatient subspecialty care for patients with AF should be considered in a dedicated trial. Consistent with this strategy, a recent large in-hospital observational registry of AF patients in North America showed that hospitalizations within 2 years after AF diagnosis were associated with increased AF symptom burden and with known limitations of contemporary drug therapy. In this analysis concerning a real-world population, the authors suggested that interventions to reduce hospitalization should reduce symptom frequency and severity and improve health-related quality of life rather than just prevent AF recurrences [28].

\section{Limitations of the study}

There are several weaknesses in our study. The results of our study have been derived from patients presenting to our tertiary care teaching hospital and need validation in other centers. Selection of patients was not randomized; the 3 groups of patients were drawn from 3 different time periods; and the emergence of the outpatient clinic, which was one modality designed to reduce admission, came at a time of pressure toward reducing admissions by the hospital's administration. Indeed, our general admission rate from the ED was about $20 \%$ of patients during the 1990 s and rose to about $25 \%$ of patients in the first years of $2000 \mathrm{~s}$. However, our tertiary care community hospital accepts patients referred from other hospitals for complications like acute coronary syndromes, pulmonary embolism, severe trauma, life-threatening tachyarrhythmias, and sepsis.

The reduction in admission frequency between the 3 time periods might not be the result of the outpatient clinic per se, but rather the result of the overall increased attention paid to admission avoidance. However, the intensive observation unit works in implementing the use of early and aggressive antiarrhythmic therapy. Moreover, the results in our cohort of patients are not extensible to the symptomatic general population because of the exclusion of patients with Killip class greater than or equal to 2; New York Heart Association functional class greater than or equal to III; complications like acute coronary syndrome, stroke, or pulmonary or peripheral embolism; and hemodynamic instability. Another possible limitation is due to the fact that, in our series, duration of AF was based on the clinical history; we aware that patients often do not feel symptoms until heart rate is rapid or pressure increases. However, our data suggest that early administration of class IC antiarrhythmic drugs has a rapid conversion rate and allows discharge from the ED. This seems to be the main finding of the manuscript and reduces the emphasis of the outpatient clinic. We did not use ibutilide. We aware that ibutilide showed good outcomes in acute AF patients. However, ibutilide is a costly drug in Italy (more than 10-fold the cost of amiodarone, propafenone, and flecainide) and has not been admitted to hospital protocol of antiarrhythmic drugs by our administration. Moreover, the need of prolonged ECG monitoring for reported ventricular tachycardia and torsades de pointes could lead to prolonged observation and costly management. Finally, the optimal use of outpatients clinic in patients with AF needs to be confirmed in a properly designed study beyond the preliminary results of this observational analysis.

\section{Conclusion}

In patients presenting with AF, beyond the standard approach, the novel organization with an additional intensive observation unit for early pharmacological interventions and an outpatient clinic for elective treatment and short-term follow-up significantly reduced admission irrespective of independent predictors of hospitalizations such as lack of 
rhythm control, comorbidities, diabetes, and age. Patients with acute AF without structural heart disease subjected to antiarrhythmic drugs achieved sinus rhythm in $89 \%$ of cases, mostly with class IC drugs, and were discharged. More than a quarter of patients were referred to the outpatient clinic for elective treatment and follow-up, avoiding admission.

\section{References}

[1] Decker WW, Smars PA, Vaidyanathan L, Goyal DG, Boie ET, Stead $\mathrm{LG}$, et al. A prospective, randomized trial of an emergency department observation unit for acute onset atrial fibrillation. Ann Emerg Med 2008;52(4):322-8

[2] Wattigney WA, Mensah GA, Croft JB. Increasing trends in hospitalization for atrial fibrillation in the United States, 1985 through 1999: implications for primary prevention. Circulation 2003;108(6): 711-6.

[3] Friberg J, Buch P, Scharling H, Gadsbphioll N, Jensen GB. Rising rates of hospital admissions for atrial fibrillation. Epidemiology 2003; 14(6):666-72.

[4] Roger VL, Go AS, Lloyd-Jones DM, Adams RJ, Berry JD, Brown TM, et al. Heart disease and stroke statistics - 2011 update: a report from the American Heart Association. Circulation 2011;123(4): e18-209.

[5] Camm AJ, Kirchhof P, Lip GY, Schotten U, Savelieva I, Ernst S, et al. Guidelines for the management of atrial fibrillation: the Task Force for the Management of Atrial Fibrillation of the European Society of Cardiology (ESC). Europace 2010;12(10):1360-420.

[6] Miyasaka Y, Barnes ME, Gersh BJ, Cha SS, Bailey KR, Seward JB, et al. Changing trends of hospital utilization in patients after their first episode of atrial fibrillation. Am J Cardiol 2008;102(5):568-72.

[7] Humphries KH, Jackevicius C, Gong Y, Svensen L, Cox J, Tu JV, et al. Population rates of hospitalization for atrial fibrillation/flutter in Canada. Can J Cardiol 2004;20(9):869-76.

[8] Reynolds MR, Essebag V, Zimetbaum P, Cohen DJ. Healthcare resource utilization and costs associated with recurrent episodes of atrial fibrillation: the FRACTAL registry. J Cardiovasc Electrophysiol 2007;18(6):628-33.

[9] Chugh SS, Blackshear JL, Shen WK, Hammill SC, Gersh BJ. Epidemiology and natural history of atrial fibrillation: clinical implications. J Am Coll Cardiol 2001;37(2):371-8.

[10] Tsang TS, Petty GW, Barnes ME, O'Fallon WM, Bailey KR, Wiebers DO, et al. The prevalence of atrial fibrillation in incident stroke cases and matched population controls in Rochester, Minnesota: changes over three decades. J Am Coll Cardiol 2003;42(1):93-100.

[11] Wann LS, Curtis AB, January CT, Ellenbogen KA, Lowe JE, Estes III NA, et al. 2011 ACCF/AHA/HRS focused update on the management of patients with atrial fibrillation (updating the 2006 guideline): a report of the American College of Cardiology Foundation/American Heart Association Task Force on Practice Guidelines. Heart Rhythm 2011;8(1):157-76

[12] Anderson JL, Adams CD, Antman EM, Bridges CR, Califf RM, Casey Jr $\mathrm{DE}$, et al. $2011 \mathrm{ACCF} / \mathrm{AHA}$ focused update incorporated into the ACC/AHA 2007 guidelines for the management of patients with unstable angina/non-ST-elevation myocardial infarction: a report of the American College of Cardiology Foundation/American Heart Association Task Force on Practice Guidelines. Circulation 2011;123(18):e426-579.

[13] Hamm CW, Bassand JP, Agewall S, Bax J, Boersma E, Bueno H. ESC guidelines for the management of acute coronary syndromes in patients presenting without persistent ST-segment elevation: the Task Force for the Management of Acute Coronary Syndromes (ACS) in Patients Presenting Without Persistent ST-Segment Elevation of the European Society of Cardiology (ESC). Eur Heart J 2011;32(23): 2999-3054.

[14] Van de Werf F, Bax J, Betriu A, Blomstrom-Lundqvist C, Crea F, Falk $\mathrm{V}$, et al. Management of acute myocardial infarction in patients presenting with persistent ST-segment elevation: the Task Force on the Management of ST-Segment Elevation Acute Myocardial Infarction of the European Society of Cardiology. Eur Heart J 2008;29(23): 2909-45.

[15] Neumar RW, Otto CW, Link MS, Kronick SL, Shuster M, Callaway CW. Part 8: adult advanced cardiovascular life support: 2010 American Heart Association guidelines for cardiopulmonary resuscitation and emergency cardiovascular care. Circulation 2010;122(18 Suppl 3): S729-67.

[16] Conti A, Del TB, Mariannini Y, Pepe G, Vanni S, Grifoni S, et al Management of patients with acute atrial fibrillation in the ED. Am J Emerg Med 2010;28(8):903-10.

[17] del AC, Martin A, Laguna P, Gargantilla P. Analysis of current management of atrial fibrillation in the acute setting: GEFAUR-1 study. Ann Emerg Med 2005;46(5):424-30.

[18] Naccarelli GV, Wolbrette DL, Khan M, Bhatta L, Hynes J, Samii S. Old and new antiarrhythmic drugs for converting and maintaining sinus rhythm in atrial fibrillation: comparative efficacy and results of trials. Am J Cardiol 2003;91(6A):15D-26D.

[19] Naccarelli GV, Wolbrette DL, Bhatta L, Khan M, Hynes J, Samii S, et al. A review of clinical trials assessing the efficacy and safety of newer antiarrhythmic drugs in atrial fibrillation. J Interv Card Electrophysiol 2003;9(2):215-22.

[20] Roberts R, Graff LG. Economic issues in observation unit medicine Emerg Med Clin North Am 2001;19(1):19-33.

[21] Farkouh ME, Smars PA, Reeder GS, Zinsmeister AR, Evans RW, Meloy TD, et al. A clinical trial of a chest-pain observation unit for patients with unstable angina. Chest Pain Evaluation in the Emergency Room (CHEER) Investigators. N Engl J Med 1998;339(26):1882-8.

[22] Rydman RJ, Isola ML, Roberts RR, Zalenski RJ, McDermott MF, Murphy DG, et al. Emergency department observation unit versus hospital inpatient care for a chronic asthmatic population: a randomized trial of health status outcome and cost. Med Care 1998; 36(4):599-609.

[23] Smars PA, Decker WW, Shen WK. Syncope evaluation in the emergency department. Curr Opin Cardiol 2007;22(1):44-8.

[24] Raghavan AV, Decker WW, Meloy TD. Management of atrial fibrillation in the emergency department. Emerg Med Clin North Am 2005;23(4):1127-39.

[25] Zimetbaum P, Reynolds MR, Ho KK, Gaziano T, McDonald MJ, McClennen S, et al. Impact of a practice guideline for patients with atrial fibrillation on medical resource utilization and costs. Am J Cardiol 2003;92(6):677-81.

[26] Nichol G, McAlister F, Pham B, Laupacis A, Shea B, Green M, et al. Meta-analysis of randomised controlled trials of the effectiveness of antiarrhythmic agents at promoting sinus rhythm in patients with atrial fibrillation. Heart 2002;87(6):535-43.

[27] Camm AJ, Capucci A, Hohnloser SH, Torp-Pedersen C, van Gelder IC, Mangal B, et al. A randomized active-controlled study comparing the efficacy and safety of vernakalant to amiodarone in recent-onset atrial fibrillation. J Am Coll Cardiol 2011;57(3):313-21.

[28] Reynolds MR, Morais E, Zimetbaum P. Impact of hospitalization on health-related quality of life in atrial fibrillation patients in Canada and the United States: results from an observational registry. Am Heart J 2010;160(4):752-8. 